\title{
A Stochastic Derivative-Free Optimization Method with Importance Sampling: Theory and Learning to Control
}

\author{
Adel Bibi, ${ }^{1}$ El Houcine Bergou, ${ }^{1,2}$ Ozan Sener, ${ }^{3}$ Bernard Ghanem, ${ }^{1}$ Peter Richtárik ${ }^{1,4}$ \\ ${ }^{1}$ King Abdullah University of Science and Technology \\ ${ }^{2}$ MaIAGE, INRA, Université Paris-Saclay \\ ${ }^{3}$ Intel Labs \\ ${ }^{4}$ Moscow Institute of Physics and Technology \\ \{adel.bibi, houcine.bergou, bernard.ghanem, peter.richtarik\}@kaust.edu.sa, ozan.sener@intel.com
}

\begin{abstract}
We consider the problem of unconstrained minimization of a smooth objective function in $\mathbb{R}^{n}$ in a setting where only function evaluations are possible. While importance sampling is one of the most popular techniques used by machine learning practitioners to accelerate the convergence of their models when applicable, there is not much existing theory for this acceleration in the derivative-free setting. In this paper, we propose the first derivative free optimization method with importance sampling and derive new improved complexity results on non-convex, convex and strongly convex functions. We conduct extensive experiments on various synthetic and real LIBSVM datasets confirming our theoretical results. We test our method on a collection of continuous control tasks on MuJoCo environments with varying difficulty. Experiments show that our algorithm is practical for high dimensional continuous control problems where importance sampling results in a significant sample complexity improvement.
\end{abstract}

\section{Introduction}

In this paper, we consider the optimization problem

$$
\min _{x \in \mathbb{R}^{n}} f(x),
$$

where $f: \mathbb{R}^{n} \rightarrow \mathbb{R}$ is a "smooth" but not necessarily convex function, bounded from below and it achieves its global minimum at some $x_{*} \in \mathbb{R}^{n}$. In particular, we enforce throughout the paper the following smoothness assumption:

Assumption 1. The objective function $f$ has coordinatewise Lipschitz gradient, with Lispchitz constants $L_{1}, \ldots, L_{n}>0$. Moreover, $f$ is bounded from below by $f\left(x_{*}\right) \in \mathbb{R}$. That is, $f$ satisfies

$$
f\left(x_{*}\right) \leq f\left(x+t e_{i}\right) \leq f(x)+\nabla_{i} f(x) t+\frac{L_{i}}{2} t^{2}
$$

for all $x \in \mathbb{R}^{n}$ and $t \in \mathbb{R}$, where $\nabla_{i} f(x)$ is the ith partial derivative of $f$ at $x$.

DFO. We consider the Derivative-Free Optimization (DFO) (Conn, Scheinberg, and Vicente 2009; Kolda, Lewis, and

Copyright (C) 2020, Association for the Advancement of Artificial Intelligence (www.aaai.org). All rights reserved.
Torczon 2003) setting. That is, we assume that the derivatives of $f$ are numerically impractical to obtain, unreliable (e.g., noisy function evaluations (Chen 2015)), or not available at all. In typical DFO applications, evaluations of $f$ are possible through runs/simulations of some black-box software only. Optimization problems of this type appear in many applications, including computational medicine (Marsden, Feinstein, and Taylor 2008), fluid-dynamics (Allaire 2001; Haslinger and Mäckinen 2003), localization (Marsden et al. 2004; 2007) and continuous control (Mania, Guy, and Recht 2018; Salimans et al. 2017).

Literature on DFO methods for solving (1) has a long history. Some of the first approaches were based on deterministic direct search (DDS) (Hooke and Jeeves 1961). Subsequently, additional variants of DDS, including randomized approaches, were proposed in (Matyas 1965; Karmanov 1974a; 1974b; Baba 1981; Dorea 1983; Sarma 1990). However, complexity bounds for deterministic direct search methods have only been established recently by the works of (Vicente 2013; Garmanjani and Vicente 2013; Konečný and Richtárik 2014; Dodangeh and Vicente 2016). Recently, complexity bounds have also been derived for randomized methods (Diniz-Ehrhardt, Martinez, and Raydan 2008; Stich, Muller, and Gartner 2013; Gratton et al. 2015). For instance, the work of (Diniz-Ehrhardt, Martinez, and Raydan 2008; Gratton et al. 2015) imposes a decrease condition on whether to accept or reject a step of a set of random directions. Moreover, (Nesterov and Spokoiny 2017; Dvurechensky, Gasnikov, and Gorbunov 2018) derived new complexity bounds for accelerated random search.

More recently, Bergou et. al. proposed a new randomized direct search method called Stochastic Three Points (STP) method. STP, in each iteration $k$, generates a random search direction $s_{k}$ according to a certain probability law, then compares the objective function at three points: current iterate $x_{k}$, a point $x_{+}=x_{k}+\alpha_{k} s_{k}$ in the direction of $s_{k}$ and a point $x_{-}=x_{k}-\alpha_{k} s_{k}$ in the direction of $-s_{k}$. The method then chooses the best of these three points as the new iterate:

$$
x_{k+1}=\arg \min \left\{f\left(x_{k}\right), f\left(x_{+}\right), f\left(x_{-}\right)\right\} .
$$

Notation: As for the notations, $\mathbb{E}[\cdot]$ denotes the expectation operator. The standard inner product is defined as $\langle x, y\rangle=x^{\top} y$. We also denote the $\ell_{1}$-norm and $\ell_{2}$-norm by 


\section{Algorithm 1 Stochastic Three Points Method with Im- portance Sampling $\left(\mathrm{STP}_{\text {IS }}\right)$}

\section{Initialization}

Choose initial iterate $x_{0} \in \mathbb{R}^{n}$, stepsize parameters $v_{1}, \ldots, v_{n}>0$ and probabilities $p_{1}, \ldots, p_{n}>0$ summing up to 1 .

For $k=0,1,2, \ldots$.

1. Select $i_{k}=i$ with probability $p_{i}>0$.

2. Choose stepsize $\alpha_{i_{k}}$ proportional to $1 / v_{i_{k}}$.

3. Let $x_{+}=x_{k}+\alpha_{k} e_{i_{k}}$ and $x_{-}=x_{k}-\alpha_{k} e_{i_{k}}$

4. $x_{k+1}=\arg \min \left\{f\left(x_{k}\right), f\left(x_{+}\right), f\left(x_{-}\right)\right\}$

$\|\cdot\|_{1}$ and $\|\cdot\|_{2}$, respectively. We define $L=\max _{i} L_{i}$ for a given sequence of scalars $L_{1}, \ldots, L_{n}$.

\section{Paper Overview and Contributions}

While importance sampling, a term that typically refers to the nonuniform sampling of random directions in stochastic algorithms, has been widely investigated in gradient based methods (Zhao and Zhang 2015; Qu, Richtárik, and Zhang 2015; Richtárik and Takáč 2016; Stich, Raj, and Jaggi 2017), to the best of our knowledge there exists no work on importance sampling in the random direct search setting. To this end, we study STP and analyze its complexity with arbitrary probabilities. In particular, we restrict the random directions to be sampled from discrete distributions, i.e., in each iteration of STP a random direction $s_{k}$ from a finite set of independent directions $\left\{b_{1}, \ldots, b_{n}\right\} \subset \mathbb{R}^{n}$ is sampled. That is, we set $s_{k}=b_{i}$ with probability $p_{i}>0$. We then propose new sampling strategies that are either optimal or at least improve the complexity bounds, i.e., importance sampling.

\section{Coordinate directions}

Without loss of generality, we only consider directions in the canonical basis of $\mathbb{R}^{n}$, i.e., $e_{1}, \ldots, e_{n}$. The general case can be recovered via a linear change of variables: $x=B y$, where $B \in \mathbb{R}^{n \times n}$. Indeed, consider the problem

$$
\min _{y \in \mathbb{R}^{n}} f_{B}(y) \stackrel{\text { def }}{=} f(B y)
$$

instead. A coordinate update $y_{k+1}=y_{k}+\alpha_{k} e_{i}$ for the re-parameterized problem (2) corresponds to updates of the form $x_{k+1}=x_{k}+\alpha_{k} b_{i}$, where $b_{i}$ is the $i$ th column of $B$, for the original problem (1). In light of the above discussion, the newly proposed algorithm dubbed $\mathrm{STP}_{\text {IS }}$ is formally described as Algorithm 1.

\section{Complexity bounds}

To the best of our knowledge, ours are the first complexity bounds (bounds on the number of iterations) for a DFO method with importance sampling. We design importance sampling that improves the worst-case iteration complexity bounds compared to state-of-the-art algorithms. These bounds have the same dependence on the precision $\epsilon$ as classical bounds in the literature, i.e. $1 / \epsilon^{2}$ for non-convex $f, 1 / \epsilon$ for convex $f$ and $\log (1 / \epsilon)$ for strongly convex $f$; see for instance (Bergou, Gorbunov, and Richtárik 2019; Nesterov and Spokoiny 2017). However, the leading constant, which is often the bottleneck in practical performance, especially when low or medium accuracy solutions are acceptable, is improved and often dramatically so. Typically, the improvement is via replacing the maximum Lipschitz constant of the gradient by the average Lipschitz constants of all coordinates (see Theorems 1, 2, 3, and $4)$. The improvement we obtain is similar to the improvement obtained by importance sampling in stochastic coordinate (gradient) descent methods (Zhao and Zhang 2015; Qu, Richtárik, and Zhang 2015; Richtárik and Takáč 2016). Table 1 summarizes complexity results obtained in this paper for STP and for $\mathrm{STP}_{\text {IS }}$. The assumptions in Table 1 are in addition to Assumption 1.

\section{Empirical results}

In addition to our theoretical analysis, we conduct extensive testing to show the efficiency of the proposed method in practice. We use both synthetic and real $^{1}$ datasets for ridge regression and squared SVM problems. In the nonconvex case, we use continuous control tasks from the $\mathrm{Mu}-$ JoCo (Todorov, Erez, and Tassa 2012) suite following the recent success of DFO compared to model-free RL (Mania, Guy, and Recht 2018; Salimans et al. 2017). Results show that our approach leads to huge speedups compared against uniform sampling, the improvement can reach several orders of magnitude and comparable or better than state-of-art policy gradient methods.

\section{Non-Convex Case}

This section describes our complexity results for Algorithm 1 in the case when $f$ is allowed to be non-convex. We show that this method guarantees complexity bounds with the same order in $\epsilon$ as classical bounds in the literature, i.e., $1 / \epsilon^{2}$ with an improved dependence on the Lipschitz constant. All proofs are left for the appendix.

Theorem 1. Let Assumption 1 be satisfied. Choose $\alpha_{i_{k}}=$ $\frac{\alpha_{0}}{v_{i_{k}} \sqrt{k+1}}$ where $\alpha_{0}>0$. If

$$
K \geq \frac{2\left(\frac{\sqrt{2}\left(f\left(x_{0}\right)-f\left(x_{*}\right)\right)}{\alpha_{0}}+\frac{\alpha_{0} \sum_{i=1}^{n} \frac{p_{i} L_{i}}{v_{i}^{2}}}{2}\right)^{2}}{\left(\min _{i} \frac{p_{i}}{v_{i}}\right)^{2} \epsilon^{2}},
$$

then $\min _{k=0,1, \ldots, K} \mathbb{E}\left[\left\|\nabla f\left(x_{k}\right)\right\|_{1}\right] \leq \epsilon$.

Note that the complexity depends on $\alpha_{0}$. The optimal choice of $\alpha_{0}$ minimizing (3) is $\alpha_{0}^{*}=8^{1 / 4} \sqrt{\frac{f\left(x_{0}\right)-f\left(x_{*}\right)}{\sum_{i=1}^{n} \frac{p_{i} L_{i}}{v_{i}^{2}}}}$,

\footnotetext{
${ }^{1}$ We use several LIBSVM datasets (Chang and Lin 2011).
} 


\begin{tabular}{c|c|c|c|c}
\hline Assumptions on $f$ & $\begin{array}{c}\text { Uniform Sampling } \\
\text { Complexity }\end{array}$ & $\begin{array}{c}\text { Importance } \\
\text { Sampling }\end{array}$ & $\begin{array}{c}\text { Importance Sampling } \\
\text { Complexity [NEW] }\end{array}$ & Theorem \\
\hline None & $\frac{4 \sqrt{2} r_{0} n^{2} L}{\epsilon^{2}}$ & $p_{i}=\frac{\sqrt{L_{i}}}{\sum_{i=1}^{n} \sqrt{L_{i}}}$ & $\frac{4 \sqrt{2} r_{0}\left(\sum_{i=1}^{n} \sqrt{L_{i}}\right)^{2}}{\epsilon^{2}}$ & 1 \\
None & $\frac{4 \sqrt{2} r_{0} n^{2} L}{\epsilon^{2}}$ & $p_{i}=\frac{L_{i}}{\sum_{i=1}^{n} L_{i}}$ & $\frac{4 \sqrt{2} r_{0} n\left(\sum_{i=1}^{n} L_{i}\right)}{\epsilon^{2}}$ & 2 \\
Convex, $R_{0}<\infty$ & $8 R_{0}^{2} n^{2} L\left(\frac{1}{\epsilon}-\frac{1}{r_{0}}\right)$ & $p_{i}=\frac{L_{i}}{\sum_{i=1}^{n} L_{i}}$ & $8 R_{0}^{2} n \sum_{i=1}^{n} L_{i}\left(\frac{1}{\epsilon}-\frac{1}{r_{0}}\right)$ & 3 \\
$\lambda$-strongly convex & $\frac{n L}{\lambda} \log \left(\frac{r_{0}}{\epsilon}\right)$ & $p_{i}=\frac{L_{i}}{\sum_{i=1}^{n} L_{i}}$ & $\frac{\sum_{i=1}^{n} L_{i}}{\lambda} \log \left(\frac{r_{0}}{\epsilon}\right)$ & 4 \\
\hline
\end{tabular}

Table 1: Summary of the new derived complexity results as opposed to uniform sampling where $r_{0}=f\left(x_{0}\right)-f\left(x_{*}\right)$. The assumptions listed are in addition to Assumption 1. $R_{0}<\infty$ indicates a bounded level set where the exact definition is given in Assumption 2. The key differences in complexity between the uniform and importance sampling are detailed in text.

in which case the complexity bound (3) takes the form

$$
\frac{4 \sqrt{2}\left(f\left(x_{0}\right)-f\left(x_{*}\right)\right) \sum_{i=1}^{n} \frac{p_{i} L_{i}}{v_{i}^{2}}}{\left(\min _{i} \frac{p_{i}}{v_{i}}\right)^{2} \epsilon^{2}} .
$$

Importance sampling. The complexity depends on the choice of the probabilities $\left\{p_{i}\right\}_{i=1}^{n}$ and the quantities $\left\{v_{i}\right\}_{i=1}^{n}$. For instance, if $p_{i}=\frac{\sqrt{L_{i}}}{\sum_{i=1}^{n} \sqrt{L_{i}}}$ and $v_{i}=\sqrt{L_{i}}$, then the complexity becomes

$$
\frac{4 \sqrt{2}\left(f\left(x_{0}\right)-f\left(x_{*}\right)\right)\left(\sum_{i=1}^{n} \sqrt{L_{i}}\right)^{2}}{\epsilon^{2}} .
$$

On the other hand, if $p_{i}=\frac{L_{i}}{\sum_{i=1}^{n} L_{i}}$ and $v_{i}=L_{i}$ then the complexity becomes

$$
\frac{4 \sqrt{2}\left(f\left(x_{0}\right)-f\left(x_{*}\right)\right) n\left(\sum_{i=1}^{n} L_{i}\right)}{\epsilon^{2}} .
$$

Under the choice of uniform sampling, i.e. $p_{i}=\frac{1}{n}$ and the choice $v_{i}=L$, we recover the uniform sampling complexity of (Bergou, Gorbunov, and Richtárik 2019)

$$
\frac{4 \sqrt{2}\left(f\left(x_{0}\right)-f\left(x_{*}\right)\right) n^{2} L}{\epsilon^{2}} .
$$

Note that $\left(\sum_{i=1}^{n} \sqrt{L_{i}}\right)^{2} \leq n^{2} L$ and $n\left(\sum_{i=1}^{n} L_{i}\right) \leq n^{2} L$. Therefore, complexity bounds in the number of iterations is improved with the proposed importance sampling strategies (4) and (5). We now state a complexity theorem for Algorithm 1 when using non-decreasing stepsizes.

Theorem 2. Let Assumption 1 be satisfied. Choose $\alpha_{i_{k}}=$ $\frac{\epsilon}{n v_{i_{k}}}$ where $\sum_{i=1}^{n} \frac{p_{i} L_{i}}{v_{i}^{2}}<2 n\left(\min _{i} \frac{p_{i}}{v_{i}}\right)$. If

$$
K \geq \frac{2 n\left(f\left(x_{0}\right)-f\left(x_{*}\right)\right)}{\left(\min _{i} \frac{p_{i}}{v_{i}}\right)\left(1-\frac{\sum_{i=1}^{n} \frac{p_{i} L_{i}}{v_{i}^{2}}}{2 n\left(\min _{i} \frac{p_{i}}{v_{i}}\right)}\right) \epsilon^{2}},
$$

then $\min _{k=0,1, \ldots, K} \mathbb{E}\left[\left\|\nabla f\left(x_{k}\right)\right\|_{1}\right] \leq \epsilon$.

Under the choice of importance sampling $p_{i}=\frac{L_{i}}{\sum_{i=1}^{n} L_{i}}$ and $v_{i}=L_{i}$, the complexity (6) becomes

$$
\frac{4\left(f\left(x_{0}\right)-f\left(x_{*}\right)\right) n\left(\sum_{i=1}^{n} L_{i}\right)}{\epsilon^{2}} .
$$

Similar to Theorem 1, the uniform sampling complexity of (Bergou, Gorbunov, and Richtárik 2019) can be recovered with $p_{i}=\frac{1}{n}$ and $v_{i}=L$. Note that the uniform sampling complexity is proportional to $n^{2} L$ and since $n \sum_{i=1}^{n} L_{i} \leq$ $n^{2} L$, the worst case complexity of the number of iterations is also improved for this choice of importance sampling.

\section{Convex Case}

This section describes our complexity results for Algorithm 1 when the objective function $f$ is convex. We show that this method guarantees complexity bounds with the same order in $\epsilon$ as classical bounds in the literature, i.e., $1 / \epsilon$ with an improved dependence on the Lipschitz constant. We will need the following additional assumption in the sequel.

Assumption 2. The function $f$ is convex and has a bounded level set at $x_{0}$. That is, $f$ satisfies:

$$
R_{0} \stackrel{\text { def }}{=} \max _{x}\left\{\left\|x-x_{*}\right\|_{\infty}: f(x) \leq f\left(x_{0}\right)\right\}<\infty
$$

Note that if $f$ is convex and has bounded level sets, the following holds:

$$
\begin{aligned}
f(x)-f\left(x_{*}\right) & \leq\left\langle\nabla f(x), x-x_{*}\right\rangle \\
& \leq\|\nabla f(x)\|_{1}\left\|x-x_{*}\right\|_{\infty} \leq R_{0}\|\nabla f(x)\|_{1} .
\end{aligned}
$$

Theorem 3. Let Assumptions 1 and 2 be satisfied. Choose $\alpha_{i_{k}}=\frac{\left|f\left(x_{k}+t e_{i_{k}}\right)-f\left(x_{k}\right)\right|}{t v_{i_{k}}}$ and sufficiently small $t$ (see the appendix for the bound on $t$ ). If

$$
k \geq \frac{8 R_{0}^{2} n}{\min _{i} \frac{p_{i}}{v_{i}}}\left(\frac{1}{\epsilon}-\frac{1}{r_{0}}\right),
$$

then $\mathbb{E}\left[f\left(x_{k}\right)-f\left(x_{*}\right)\right] \leq \epsilon$.

Here, the complexity bound in (9) depends on the choice of the probabilities $\left\{p_{i}\right\}_{i=1}^{n}$ and the quantities $\left\{v_{i}\right\}_{i=1}^{n}$. Note that if $v_{i}=L_{i}$, it is easy to show that the minimum of the complexity bound (9) in $p_{i}$, i.e. minimizing $1 / \min _{i} \frac{p_{i}}{v_{i}}$ in $p_{i}$, over a probability simplex is attained at $p_{i}=\frac{L_{i}}{\sum_{i=1}^{n} L_{i}}$. Thus, the complexity bound of this this importance sampling is

$$
k \geq 8 R_{0}^{2} n\left(\sum_{i=1}^{n} L_{i}\right)\left(\frac{1}{\epsilon}-\frac{1}{r_{0}}\right) .
$$



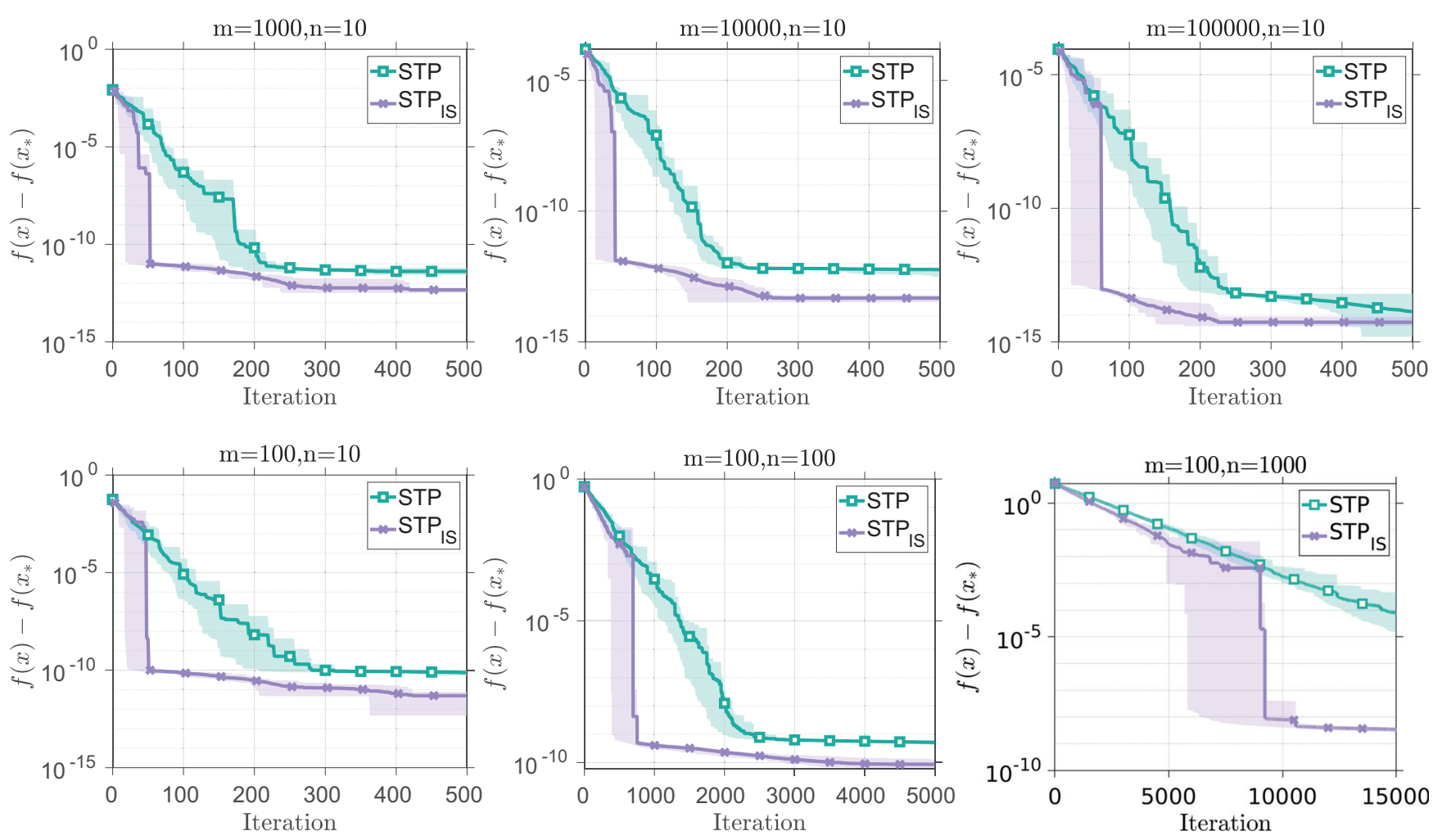

Figure 1: Shows the superiority of $\mathrm{STP}_{\text {IS }}$ over STP on synthetically generated data $A$ and $y$ on the ridge regression problem. The first row shows the comparison with a varying number of rows in $A$, i.e. $m$, while the second row shows the comparison with a varying dimension $n$.

Since uniform sampling is proportional to $n^{2} L$ and that $n \sum_{i=1}^{n} L_{i} \leq n^{2} L$, importance sampling is clearly better than uniform sampling.

\section{Strongly Convex Case}

This section describes our complexity results for Algorithm 1 when objective function $f$ is $\lambda$-strongly convex. We show that this method guarantees complexity bounds with the same order in $\epsilon$ as classical bounds in the literature, i.e., $\log (1 / \epsilon)$ with an improved dependence on the Lipschitz constant. First, we define $\lambda$-strongly convexity functions.

Assumption 3. The function $f$ is $\lambda$-strongly convex. That is, for some $\lambda>0$, the following holds

$$
f(x) \geq f(y)+\langle\nabla f(y), x-y\rangle+\frac{\lambda}{2}\|x-y\|_{2}^{2} .
$$

Theorem 4. Let Assumptions 1 and 3 be satisfied. Choose $\alpha_{i_{k}}=\frac{\left|f\left(x_{k}+t e_{i_{k}}\right)-f\left(x_{k}\right)\right|}{t v_{i_{k}}}$ and a sufficiently small $t$ (see the appendix for the bound on $t$ ). If

$$
k \geq \frac{\max _{i} \frac{v_{i}}{p_{i}}}{\lambda} \log \left(\frac{2\left(f\left(x_{0}\right)-f\left(x_{*}\right)\right)}{\epsilon}\right),
$$

then $\mathbb{E}\left[f\left(x_{k}\right)-f\left(x_{*}\right)\right] \leq \epsilon$.

The complexity bound in (10) is minimized, in $p_{i}$ for $v_{i}=$ $L_{i}$, with $p_{i}=\frac{L_{i}}{\sum_{i=1}^{n} L_{i}}$. Importance sampling improves over uniform sampling, since $\sum_{i=1}^{n} L_{i} \leq n L$.

\section{Experiments}

We conduct extensive experiments on synthetic and real datasets comparing the uniform sampling STP against the importance sampling version $\mathrm{STP}_{\mathrm{IS}}$. The experiments are conducted on several choices of the function $f$. In particular, we perform experiments on regularized ridge regression on synthetic data and squared SVM loss on real data from the LIBSVM dataset (Chang and Lin 2011). Moreover, for non-convex problems, we compare STP and $\mathrm{STP}_{\text {IS }}$ on various continuous control environments on MuJoCo (Todorov, Erez, and Tassa 2012). We also compare against state-of-art solvers for the continuous control task.

\section{Ridge regression on synthetic data}

We compare STP against $\mathrm{STP}_{\text {IS }}$ on synthetic data on the regularized ridge regression problem: $f(x)=\frac{1}{2 m} \| A x-$ $y\left\|_{2}^{2}+\frac{\lambda}{2}\right\| x \|_{2}^{2}$, where $A \in \mathbb{R}^{m \times n}, y \in \mathbb{R}^{m}$ are the data and $\lambda>0$ is the regularization parameter. The elements of $A$ and $y$ were sampled from the standard Gaussian distribution $\mathcal{N}(0,1)$. Note that for ridge regression, $L_{i}=\frac{1}{m} \| A($ : ,i) $\|_{2}^{2}+\lambda$ where, following (Gower, Richtárik, and Bach 2018), we normalize data such that $\|A(:, 1)\|_{2}=1$ and $\|A(:, i)\|_{2}=\frac{1}{m}, i=2, \ldots, m$ and set $\lambda=\frac{1}{m}$. We compute a high accuracy solution $x_{*}$ by solving the ridge regression problem exactly with a linear solver. Thereafter, the metric used is the difference between the current objective value 

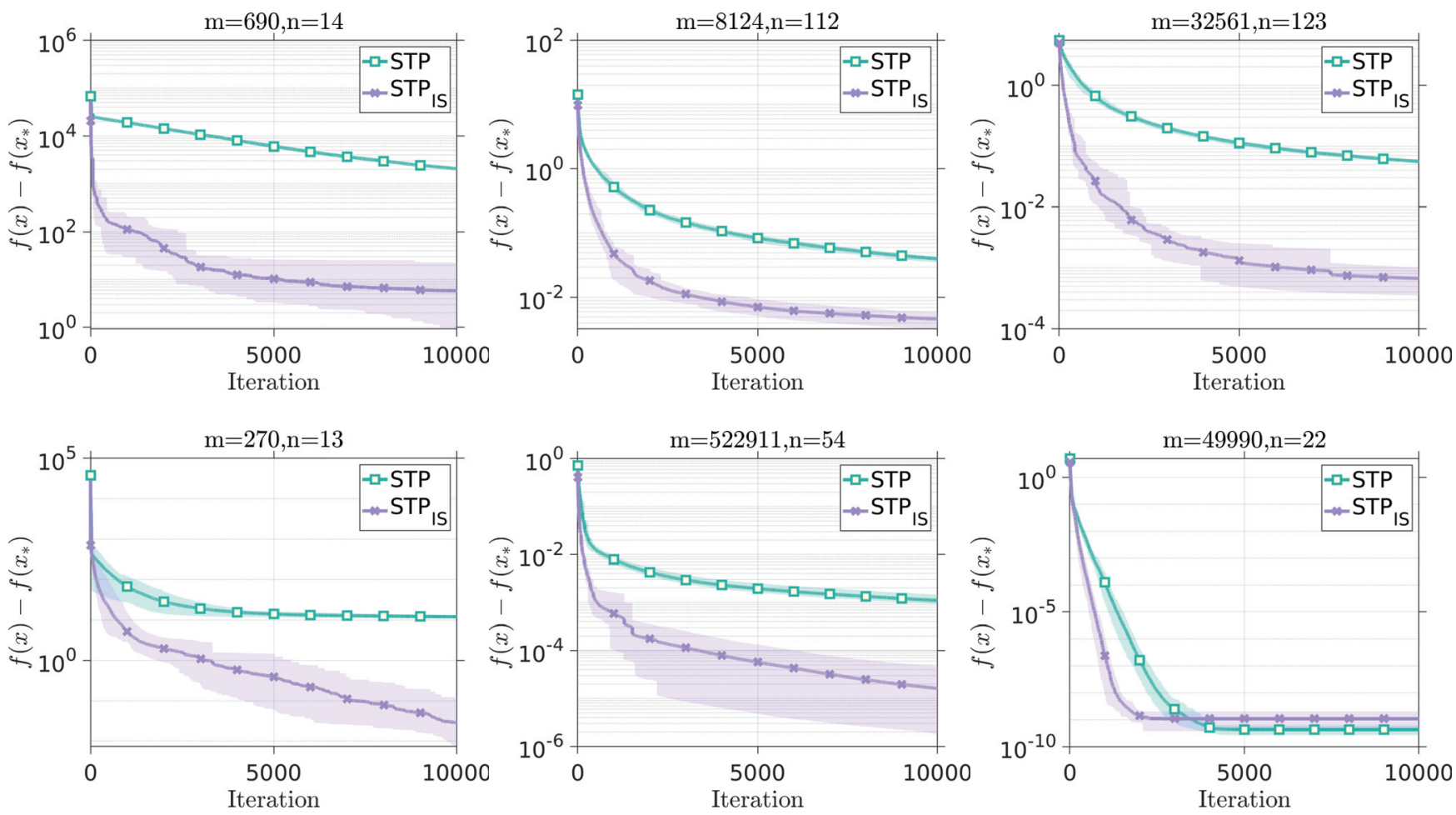

Figure 2: Shows the superiority of $\mathrm{STP}_{\text {IS }}$ over STP on real LIBSVM dataset on the ridge regression problem. The datasets used in the experiments are australian, mushrooms and a $9 \mathrm{a}$ in first row and heart, cov 1 and $i j c n n 1$ in second row.

and the optimal one, i.e. $f(x)-f\left(x_{*}\right)$. Since the objective is $\lambda$-strongly convex, we use the stepsize suggested by Theorem 4 . In all experiments, we set $t=10^{-4}$. We perform experiments across difference choices of $m$ and $n$. In the first row of Figure 1, we compare both methods with a fixed $n=10$ and a varying $m$, i.e. $m \in\left\{10^{3}, 10^{4}, 10^{5}\right\}$. The superior performance of $\mathrm{STP}_{\text {IS }}$ over $\mathrm{STP}$ is evident from Figure 1. Moreover, we conduct further experiments where $m$ is fixed such that $m=100$ but with a varying dimension, i.e. $n \in\left\{10^{1}, 10^{2}, 10^{3}\right\}$. All experiments are conducted 10 times and we report the average, worst and best performances. A similar behaviour is also present as seen in the second row of Figure 1 where $\mathrm{STP}_{\text {IS }}$ is far more superior to STP. In all experiments, the stopping criterion is set such that both STP and $\mathrm{STP}_{\text {IS }}$ run for exactly $5 \times 10^{2}$ iterations for small problems, i.e. $n=10$, while for problems of size $n=10^{2}$ and $n=10^{3}$, both methods are terminated at $5 \times 10^{3}$ and $15 \times 10^{3}$ iterations, respectively.

\section{Ridge regression and squared SVM on real data}

We also conduct experiments on the regularized ridge regression problem on real datasets where $A$ and $y$ are from LIBSVM data. We follow the same protocol as the experiments on synthetic data. We compare both algorithms on 6 different datasets, namely, australian, mushrooms, a9a, heart, covl and ijcnn1. In addition to ridge regression, we conduct experiments on the same real datasets on the regularized squared SVM loss: $f(x)=\frac{1}{2} \sum_{i=1}^{m} \max \left(0,1-y_{i} a_{i}^{\top} x\right)^{2}+\frac{\lambda}{2}\|x\|_{2}^{2}$, where $a_{i}$ is the $i^{\text {th }}$ row of $A$. Note that $L_{i}=\|A(:, i)\|_{2}^{2}+\lambda$. Since the squared SVM problem does not exhibit a closed form solution, we compare both STP and $\mathrm{STP}_{\text {IS }}$ in terms of the objective value $f(x)$. In Figure 2, we show the comparison between both $\mathrm{STP}$ and $\mathrm{STP}_{\text {IS }}$ on the ridge regression on all 6 datasets. It is clear that using the proposed importance sampling is far more superior to standard uniform sampling. The improvement is also consistently present on the squared SVM problem as seen in Figure 3.

\section{Continuous control experiments}

Here, we address the problem of model-free control of a dynamical system. Model-free reinforcement learning algorithms (especially policy gradient methods), provide an off-the-shelf model-free approach to learn how to control a dynamical system. Such models have been typically benchmarked in a simulator. Thus, we adopt the MuJoCo (Todorov, Erez, and Tassa 2012) continuous control suite following its wide adaptation. We choose 5 problems with various difficulty Swimmer-v1, Hopper-v1, HalfCheetah-v1, Ant-v1, and Humanoid-v1. In all experiments, we use linear policies similar to (Mania, Guy, and Recht 2018; Rajeswaran et al. 2017).

Considering the stochastic nature of the dynamical systems, i.e. $f$ is stochastic, we take multiple $(K)$ measurements for $f\left(x_{k}\right), f\left(x_{+}\right)$and $f\left(x_{-}\right)$and use their mean as the function values. Considering the varying dimensionality of the state space, we use different $K$ for each prob- 

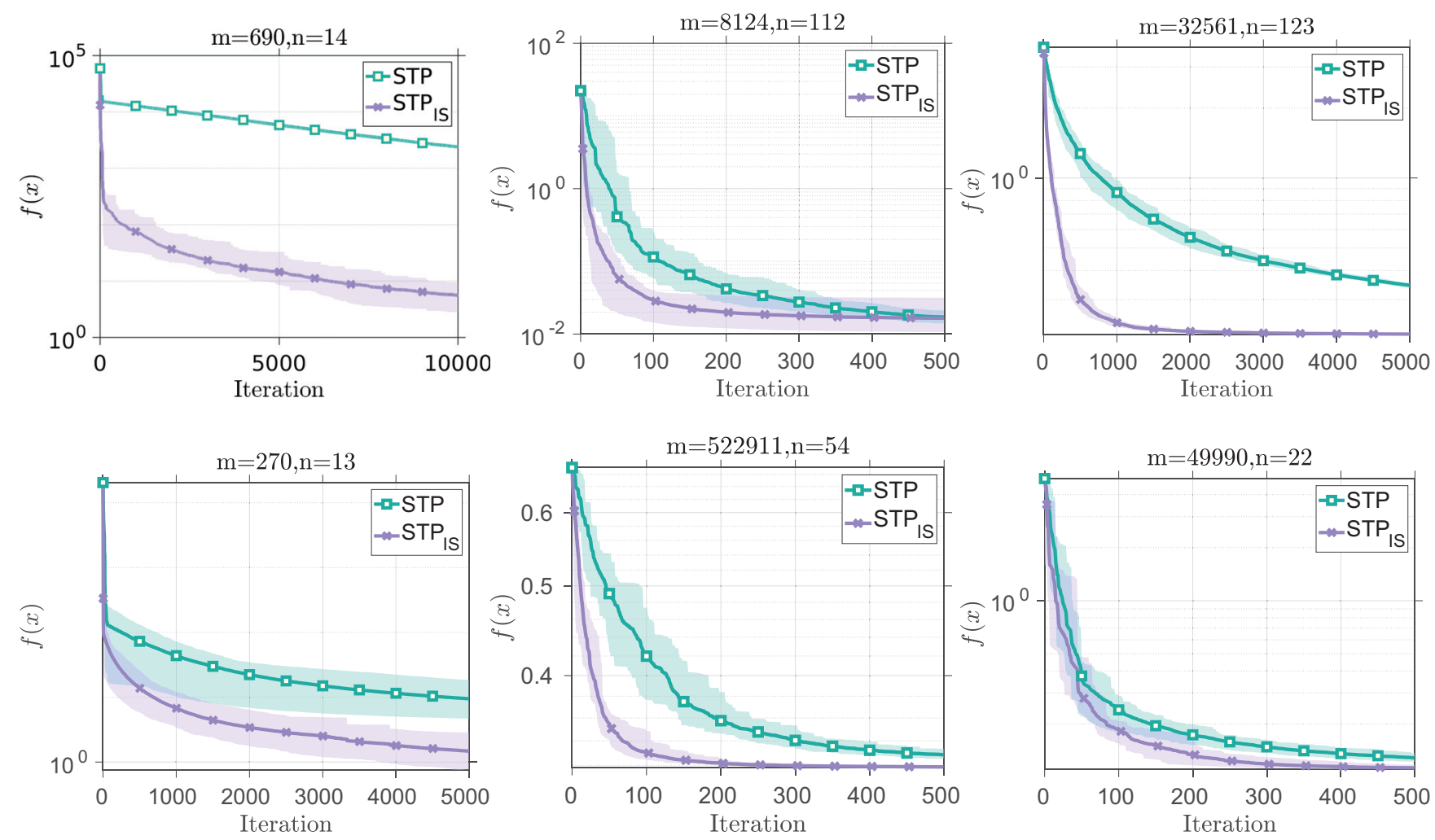

Figure 3: Shows the superiority of $\mathrm{STP}_{\text {IS }}$ over STP on real LIBSVM dataset on the squared SVM loss. The datasets used in the experiments are australian, mushrooms and a 9 a in the first row and heart, cov 1 and $i$ jenn 1 for the second row.

lem, in particular, we set $K=2$ for Swimmer-v1, $K=4$ for Hopper-v1 and HalfCheetah-v1, $K=$ 40 for Ant-v1 and $K=120$ for Humanoid-v1. These values are decided using grid search over the set of $K \in\{1,2,4,8,16\}$ for low dimensional problems and $K \in\{20,40,120,240\}$ for high dimensional Ant-v1 and Humanoid-v1 problems. Following our remark given by Equation 2, we use a square matrix $B$ sampled from a standard Gaussian distribution $\mathcal{N}(0,1)$. In our experiments, this coordinate transform resulted in a better performance. Since the Lipschitz constants are not available for continuous control, we learn an estimate of the function using a parametric family (specifically multi-layer perceptron) and use its Lipschitz constants as the estimates to decide importance sampling weights. This is similar to actor-critic methods(Sutton and Barto 1988) used in the policy gradient literature. Similar to us, actor-critic methods learn an estimate of the value function and use it to decide which point to evaluate. We defer the details of estimation procedure to the appendix. Following the common practice, we perform all experiments with 5 random initialization and measure the mean average reward at each iteration. We give detailed comparison of STP and our proposed importance sampling variant $\mathrm{STP}_{\text {IS }}$ in terms of reward vs. sample complexity in Figure 4 for both adaptive and fixed step size cases (see Theorems 1 and 2). Shaded regions in figures show standard deviations.

As seen from Figure 4, our proposed importance sam- pling version $\mathrm{STP}_{\text {IS }}$ significantly improves sample complexity when compared to STP. Moreover, the difference is significant for high dimensional problems like HalfCheetah, Ant and Humanoid. The results suggest that STP fails to scale to very high dimensional problems like Humanoid. Our method tackles this and improves the sample complexity of STP. Such results also suggest that it is feasible to estimate the coordinate-wise Lipschitz gradient constants, detailed in Assumption 1, of a complicated non-convex function using a data-driven approach. An interesting conclusion from Figure 4 is that adaptive step size performs better than fixed step size even after a large hyper-parameter search, particularly, for higher dimensional problems.

In order to compare our method with the existing state-ofart DFO and policy gradient methods, we also tabulate the sample complexity of our method and several existing baselines. Similar to (Mania, Guy, and Recht 2018), we compute the average number of episodes needed to reach a predefined threshold. Although there are many DFO and policy gradient methods in literature, we report ARS (Mania, Guy, and Recht 2018) as a representative DFO method since it outperforms other baselines. As for policy gradient approaches, we report TRPO (Schulman et al. 2015) as a representative policy gradient method since it is widely used in the community. Moreover, we use NG (Rajeswaran et al. 2017) as a policy gradient method using linear policies. We list sample complexity results for all methods and tasks in Table 2. 

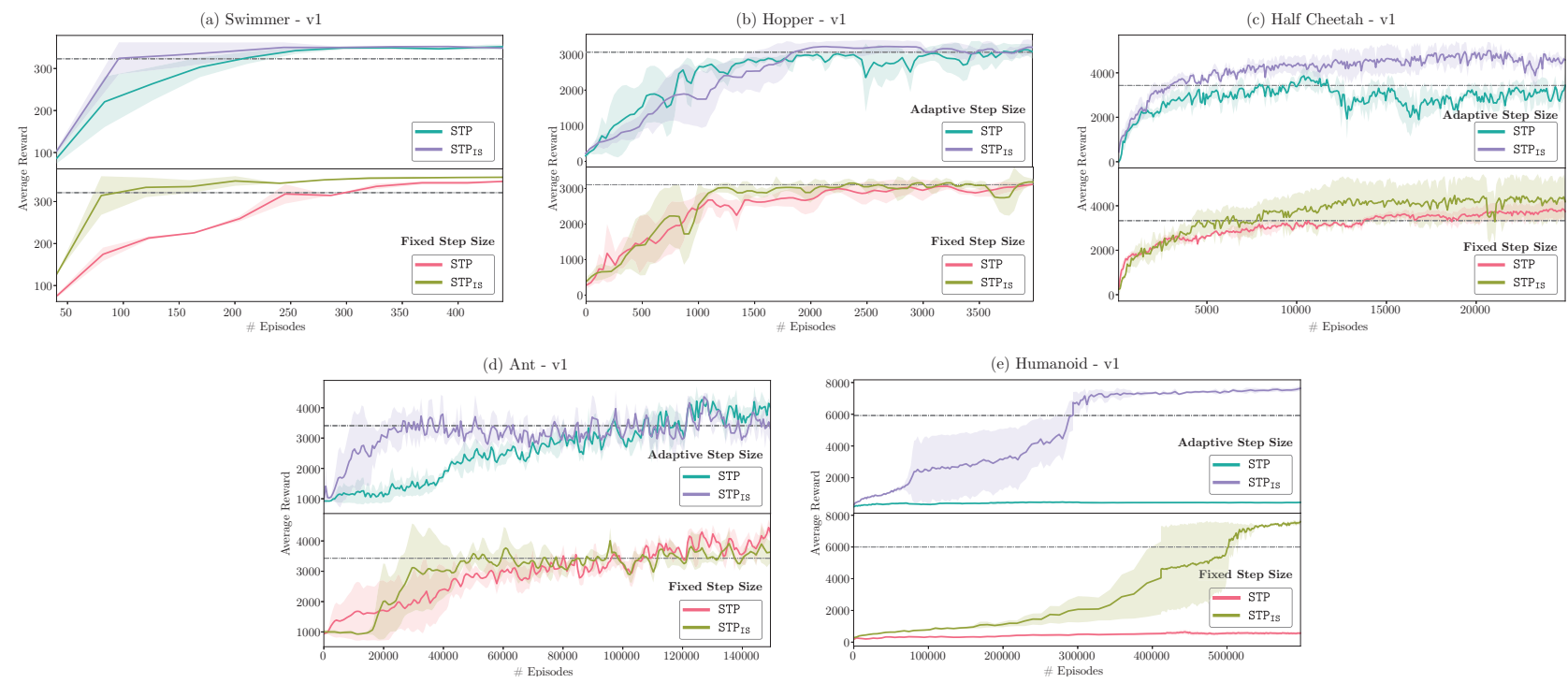

Figure 4: $\mathrm{STP}_{\text {IS }}$ is superior to STP on all 5 different MuJoCo tasks. Solid plots are the average performance over 5 runs while the shaded region shows the standard deviation. Dashed horizontal lines are the thresholds used in Table 2 to quantify sample complexity of each method.

Table 2: For each MuJoCo task, we report the average number of episodes required to achieve a predefined reward threshold. Results for our method is averaged over five random seeds, the rest is copied from (Mania, Guy, and Recht 2018) (N/A means the method failed to reach the threshold. UNK means the results is unknown since they are not reported in the literature.)

\begin{tabular}{|c|c|c|c|c|c|c|c|c|c|}
\hline & \multirow[b]{2}{*}{ Threshold } & \multicolumn{2}{|c|}{ Fixed Step Size } & \multicolumn{2}{|c|}{ Adaptive Step Size } & \multirow[b]{2}{*}{$\operatorname{ARS}(\mathrm{V} 1-\mathrm{t})$} & \multirow[b]{2}{*}{$\operatorname{ARS}(V 2-t)$} & \multirow[b]{2}{*}{ NG-lin } & \multirow[b]{2}{*}{ TRPO-nn } \\
\hline & & STP & $\mathrm{STP}_{\text {IS }}$ & STP & $\mathrm{STP}_{\text {IS }}$ & & & & \\
\hline Swimmer-v1 & 325 & 320 & 110 & 200 & 90 & 100 & 427 & 1450 & N/A \\
\hline Hopper-v1 & 3120 & 3970 & 2400 & 3720 & 1870 & 51840 & 1973 & 13920 & 10000 \\
\hline HalfCheetah-v1 & 3430 & 13760 & 4420 & 5040 & 2710 & 8106 & 1707 & 11250 & 4250 \\
\hline Ant $-\mathrm{v} 1$ & 3580 & 107220 & 43860 & 96980 & 26480 & 58133 & 20800 & 39240 & 73500 \\
\hline Humanoid-v1 & 6000 & N/A & 530200 & N/A & 296800 & N/A & 142600 & 130000 & UNK \\
\hline
\end{tabular}

As the results suggest, STP is competitive with existing solutions for low dimensional problems (Swimmer, Hopper and HalfCheetah) whereas it underperforms existing solutions for Ant and fails to solve the Humanoid problem. Our theoretically proposed importance sampling version $\mathrm{STP}_{\text {IS }}$ significantly improves STP and results in a performance either competitive with or better than existing baselines in all problems except for Humanoid. Although our method successfully solves the Humanoid problem, it has worse sample complexity than other solutions. Hence, scaling STP to very large dimensional continuous control problems (e.g. Humanoid-v1 state space has more than 1000 dimensions) is still an open problem. Moreover, for lower dimensional problems like (Swimmer and Hopper), our method outperforms all existing methods.

An interesting question is whether we can use first order methods utilizing the estimated function. In order to estimate the Lipschitz smoothness constant of the function, we utilize a parametric family and one can argue that its gradients can be used as a surrogate gradient in first order optimization. We compare our method with a simple first order baseline using the surrogate gradient in SGD; it fails in all environments. Hence, we do not show the results in the paper.

\section{Conclusion}

We propose and analyze a DFO algorithm with importance sampling $\mathrm{STP}_{\text {IS }}$ enjoying the best known complexity bounds known in DFO literature. Experiments on ridge regression and squared SVM objectives for both synthetic and LIBSVM datasets demonstrate the superiority of $\mathrm{STP}_{\text {IS }}$ over its uniform version. We also conduct experiments on a collection of continuous control tasks on several MuJoCO environments. We are orders of magnitudes better than the uniform sampling and comparable or better than the stateof-art methods.

Acknowledgments. This work was supported by the King Abdullah University of Science and Technology (KAUST) Office of Sponsored Research. 


\section{References}

Allaire, G. 2001. Shape Optimization by the Homogenization Method. New York, USA: Springer.

Baba, N. 1981. Convergence of a random optimization method for constrained optimization problems. Journal of Optimization Theory and Applications.

Bergou, E. H.; Gorbunov, E.; and Richtárik, P. 2019. Stochastic three points method for unconstrained smooth minimization. arXiv preprint arXiv:1902.03591.

Chang, C.-C., and Lin, C.-J. 2011. Libsvm: a library for support vector machines. ACM transactions on intelligent systems and technology (TIST).

Chen, R. 2015. Stochastic derivative-free optimization of noisy functions. PhD thesis at Lehigh University.

Conn, A. R.; Scheinberg, K.; and Vicente, L. N. 2009. Introduction to Derivative-Free Optimization. SIAM.

Diniz-Ehrhardt, M. A.; Martinez, J. M.; and Raydan, M. 2008. A derivative-free nonmonotone line-search technique for unconstrained optimization. Journal of Optimization Theory and Applications.

Dodangeh, M., and Vicente, L. N. 2016. Worst case complexity of direct search. Mathematical Programming.

Dorea, C. 1983. Expected number of steps of a random optimization method. Journal of Optimization Theory and Applications.

Dvurechensky, P.; Gasnikov, A.; and Gorbunov, E. 2018. An accelerated method for derivative-free smooth stochastic convex optimization. arXiv:1802.09022.

Garmanjani, R., and Vicente, L. N. 2013. Smoothing and worst-case complexity for direct-search methods in nonsmooth optimization. IMA Journal of Numerical Analysis.

Gower, R. M.; Richtárik, P.; and Bach, F. 2018. Stochastic quasi-gradient methods: Variance reduction via jacobian sketching. arXiv:1805.02632.

Gratton, S.; Royer, C. W.; Vicente, L. N.; and Zhang, Z. 2015. Direct search based on probabilistic descent. SIAM Journal on Optimization.

Haslinger, J., and Mäckinen, R. 2003. Introduction to Shape Optimization: Theory, Approximation, and Computation. SIAM.

Hooke, R., and Jeeves, T. 1961. Direct search solution of numerical and statistical problems. J. Assoc. Comput. Mach.

Karmanov, V. G. 1974a. Convergence estimates for iterative minimization methods. USSR Computational Mathematics and Mathematical Physics.

Karmanov, V. G. 1974b. On convergence of a random search method in convex minimization problems. Theory of Probability and its applications.

Kolda, T. G.; Lewis, R. M.; and Torczon, V. J. 2003. Optimization by direct search: New perspectives on some classical and modern methods. SIAM Review.

Konečný, J., and Richtárik, P. 2014. Simple complexity analysis of simplified direct search. arXiv:1410.0390.
Mania, H.; Guy, A.; and Recht, B. 2018. Simple random search provides a competitive approach to reinforcement learning. arXiv:1803.07055.

Marsden, A. L.; Wang, M.; Dennis, J. E.; and Moin, P. 2004. Optimal aeroacustic shape design using the surrogate management framework. Optimization and Engineering.

Marsden, A. L.; Wang, M.; Dennis, J. E.; and Moin, P. 2007. Trailing-edge noise reduction using derivative-free optimization and large-eddy simulation. Journal of Fluid Mechanics.

Marsden, A. L.; Feinstein, J. A.; and Taylor, C. A. 2008. A computational framework for derivative-free optimization of cardiovascular geometries. Computer Methods in Applied Mechanics and Engineering.

Matyas, J. 1965. Random optimization. Automation and Remote Control.

Nesterov, Y., and Spokoiny, V. 2017. Random gradient-free minimization of convex functions. Foundations of Computational Mathematics.

Qu, Z.; Richtárik, P.; and Zhang, T. 2015. Quartz: Randomized dual coordinate ascent with arbitrary sampling. In Advances in neural information processing systems.

Rajeswaran, A.; Lowrey, K.; Todorov, E. V.; and Kakade, S. M. 2017. Towards generalization and simplicity in continuous control. In Advances in Neural Information Processing Systems (NeurIPs).

Richtárik, P., and Takáč, M. 2016. On optimal probabilities in stochastic coordinate descent methods. Optimization Letters.

Salimans, T.; Ho, J.; Chen, X.; Sidor, S.; and Sutskever, I. 2017. Evolution strategies as a scalable alternative to reinforcement learning. arXiv:1703.03864.

Sarma, M. 1990. On the convergence of the Baba and Dorea random optimization methods. Journal of Optimization Theory and Applications.

Schulman, J.; Levine, S.; Abbeel, P.; Jordan, M.; and Moritz, P. 2015. Trust region policy optimization. In International Conference on Machine Learning (ICML).

Stich, S. U.; Muller, C. L.; and Gartner, B. 2013. Optimization of convex functions with random pursuit. SIAM Journal on Optimization.

Stich, S. U.; Raj, A.; and Jaggi, M. 2017. Safe adaptive importance sampling. Advances in Neural Information Processing Systems (NeurIPs.

Sutton, R. S., and Barto, A. G. 1988. Reinforcement learning: An introduction. IEEE Transactions on Neural Networks.

Todorov, E.; Erez, T.; and Tassa, Y. 2012. Mujoco: A physics engine for model-based control. In Intelligent Robots and Systems (IROS).

Vicente, L. N. 2013. Worst case complexity of direct search. EURO Journal on Computational Optimization.

Zhao, P., and Zhang, T. 2015. Stochastic optimization with importance sampling for regularized loss minimization. In International Conference on Machine Learning (ICML). 